\title{
General Collective Intelligence as Biomimicry of Nature's Design and Manufacturing Processes
}

\author{
Andy E. Williams, Nobeah Foundation, Nairobi, Kenya
}

\begin{abstract}
This paper explores the current limits to the complexity of design and manufacturing processes that can be reliably created or executed by humans, as well as exploring the general categories of products that can't currently can't reliably be manufactured as a consequence. This paper also explores how nature removes those barriers to complexity in its design and manufacturing, as well as how nature removes the barriers to the complex cooperation through which this complex design and manufacturing become sustainably viable, and how General Collective Intelligence replicates the adaptive problem solving processes by which nature does so. Finally, this paper explores why the type of products that can't currently be manufactured, and why the patterns of complex cooperation that can't currently be reliably executed, are both critically important, and therefore why General Collective Intelligence is critically important to the future of manufacturing.
\end{abstract}

\section{Keywords}

General Collective Intelligence, GCI, manufacturing complexity, complex manufacturing cooperation

\section{Introduction}

General Collective Intelligence or GCI is defined as a system that organizes a group into a collective intelligence with general problem solving ability that is an emergent property of the GCI. As opposed to the general problem solving ability of any individual in the group, which is intrinsically aligned with that individual's interests, the general problem-solving ability is intrinsically aligned with the group itself. GCI removes the barriers to more complex interactions within groups, where those barriers must be overcome in order to even conceive of the existence of certain collective problems or to conceive of their solutions. Without GCI, where problems face hidden barriers of complexity that make them undetectable with current group decision-making systems, or where solutions face hidden barriers that make them undiscoverable with current group decision-making systems, there are effectively hidden bugs in our decision-making that might constrain groups to using the wrong solutions to solve the wrong problems [1].

In manufacturing, General Collective Intelligence is intended to copy nature's ability to reliably create and execute the manufacturing processes required to better solve collective challenges, even where they are too complex to be created through current human manufacturing equipment and processes.

The GCI approach relies on defining functional models for design, manufacturing, logistics, planning, operations, and other processes that a business or group of businesses might engage in to achieve some outcome, where those models are universal, resulting in the potential to create value through the collective creation and exchange of these models. If one is trying to achieve an outcome in a system consisting of a business or group of businesses, in the functional modeling approach that business or group of businesses can be represented as having a set of functions. Any problem involving that system then becomes a matter of discovering to use those functions to get the system from one state, to another state. Functional models are also defined to represent products that might be produced or consumed by manufacturing or other processes of that system. These models are human-centric in being decomposable into a set of basic functions that are universally understandable to all humans without requiring any information (such as technical jargon) external to those functions. 
From this perspective any products or services, whether Internet of Things (IoT) devices, cloud based technologies, the smart grid, and other technologies that might be equipment used to automate parts of processes, or that might be produced or consumed by manufacturing or other processes, are implementations of functional models. GCI is intended to remove the barriers to using a library of processes and their functional models, and is intended to remove the barriers to using a library of products and equipment and their functional models, to increase the general problem-solving ability of the group, and therefore the ability to solve any complex manufacturing or manufacturing cooperation problem required to reach any manufacturing or manufacturing industry viability outcome.

Representing all processes involved in manufacturing or related activities through a set of functional models [1], and representing all products or equipment through a set of functional models [P], GCI seeks to solve the problem of optimizing collective outcomes achieved through all processes, in this case through all manufacturing processes.

\section{The Case of Closed Manufacturing Problems}

One category of manufacturing problems are those that are closed in that they target a specific outcome.

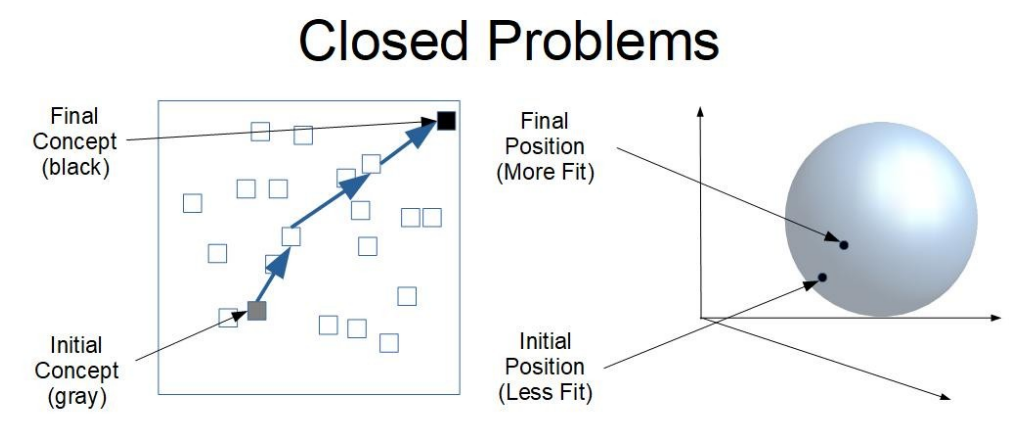

Figure 1: Closed problems can be defined in terms of any path from one concept (one point in conceptual space) to another concept, where that navigation results in changing a particular aspect of fitness.

Another are manufacturing problems that have some degree of openness in being restricted not to specific outcomes, but only to categories of outcome, where a collective intelligence evaluates projected outcomes, or outcomes actually achieved, in terms of impact of those outcomes on overall fitness to execute all functions. Only closed manufacturing problems are considered here. 


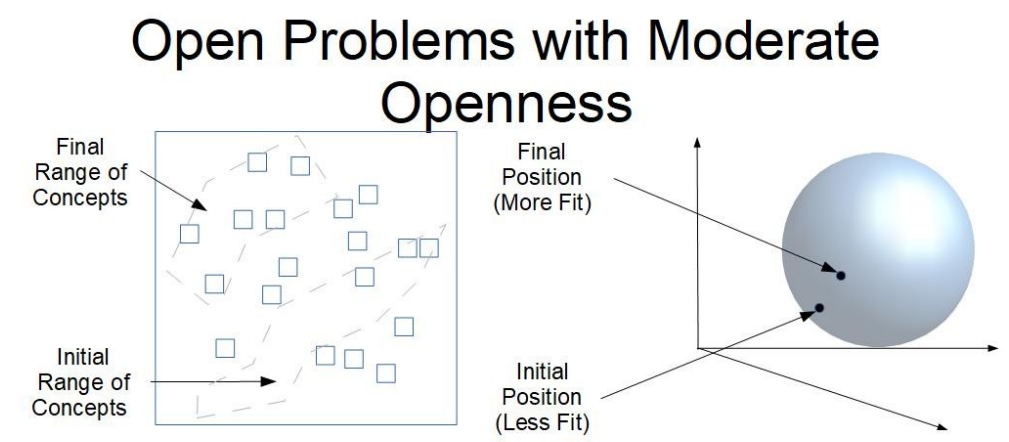

Figure 2: Problems with moderate openness can potentially be defined in terms of any path from any one in a set of concepts (one region in conceptual space) to any other in another set of concepts, where that navigation results in changing a particular aspect of fitness.

Having defined a simple functional model of each product and process, this approach then uses functional decomposition to break the each down into a number of functional components to remove the barriers to massive collaboration by enabling anyone to replace any functional model of a component or the entire system of manufacturing processes itself, to replace any implementation of any functional model, or to replace anything else, by something that is more fit at achieving the targeted goals.

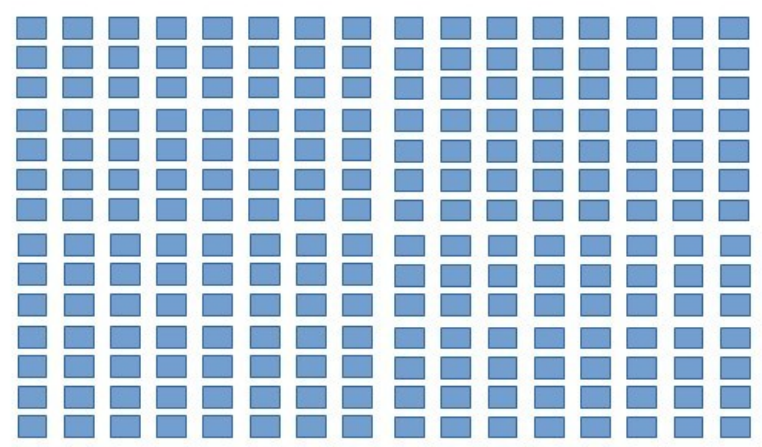

Figure 3 Functional components involved in overall manufacturing process

In order to create a complete manufacturing process on their own, any product or services provider would potentially have to implement all of these functionalities, or find an implementation that was compatible with their approach. Having done so they would also have to ensure deployment at scale so there are enough users to ensure implementing all the functionality required to achieve their goals is economically feasible. However, through collectively intelligent cooperation, each manufacturer could significantly reduce its work by limiting itself to having to develop only a restricted set of processes. 
The goal of orchestrating such cooperation is not creating all these processes. Instead it's to define the process components required to solve manufacturing problems, and to identify the components in each manufacturer's processes that might serve as solutions, so that the manufacturing problems and solutions can be matched together. Or alternatively, to coordinate with Prospective Partners who are investing in creating such manufacturing processes, so that rather than them funding the implementation of all processes and having a high likelihood of not being successful at implementing any where those processes are too complex, each participant can agree to invest in some subset so that together there is a high likelihood of being successful at all. Through this cooperation all parties might increase their impact on their targeted goals.

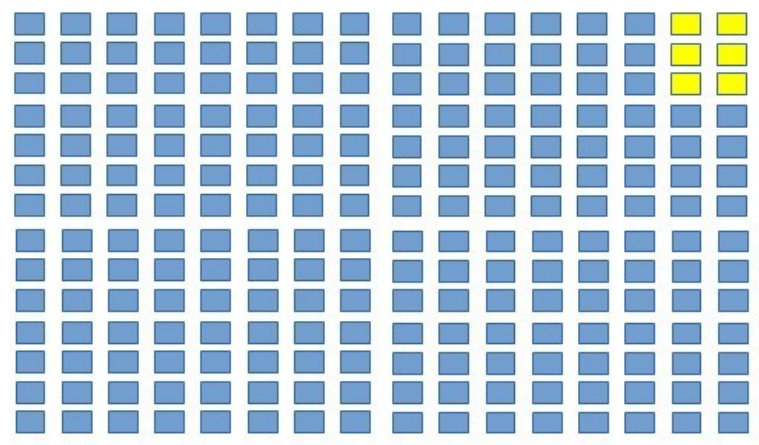

Figure 5 Map the Functional components in each vendor or project's product or service to the overall problem

Creating functional models of each organization's manufacturing processes, and defining general processes for assembling those processes, enables algorithms to be defined to computationally find optimal matches between problems and solutions at orders of magnitude greater speed and scale.

\section{- Problems}

- Solutions
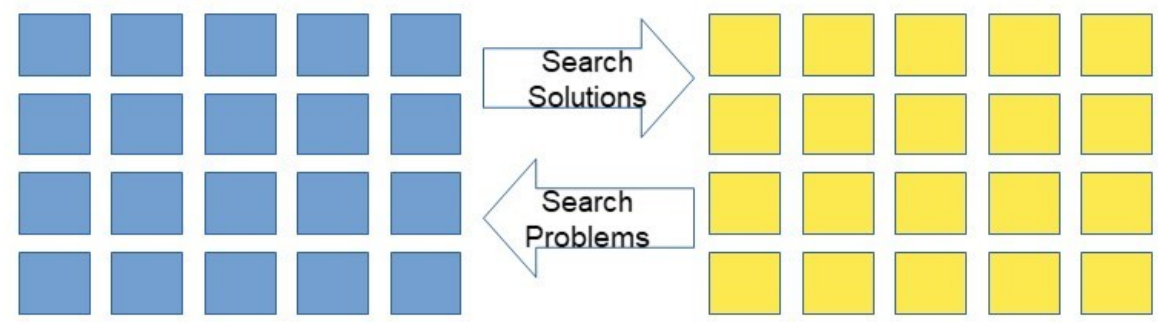

Figure 6: Using algorithms to computationally find optimal matches between problems and solutions at orders of magnitude greater speed and scale.

In this human-centric approach to functional modeling of physical or virtual processes, each function a solution is required to have is represented in terms of an input, an output, a context of execution, and an outcome related to that output. In the case of virtual processes, the context of execution represents all the information that the output is dependent on that is not part of the input, such as configuration parameters. This modeling is human-centric in that the inputs, outputs, and context are concepts or sets of concepts that are defined semantically. An approach to representing these entities semantically is presented in detail elsewhere. 


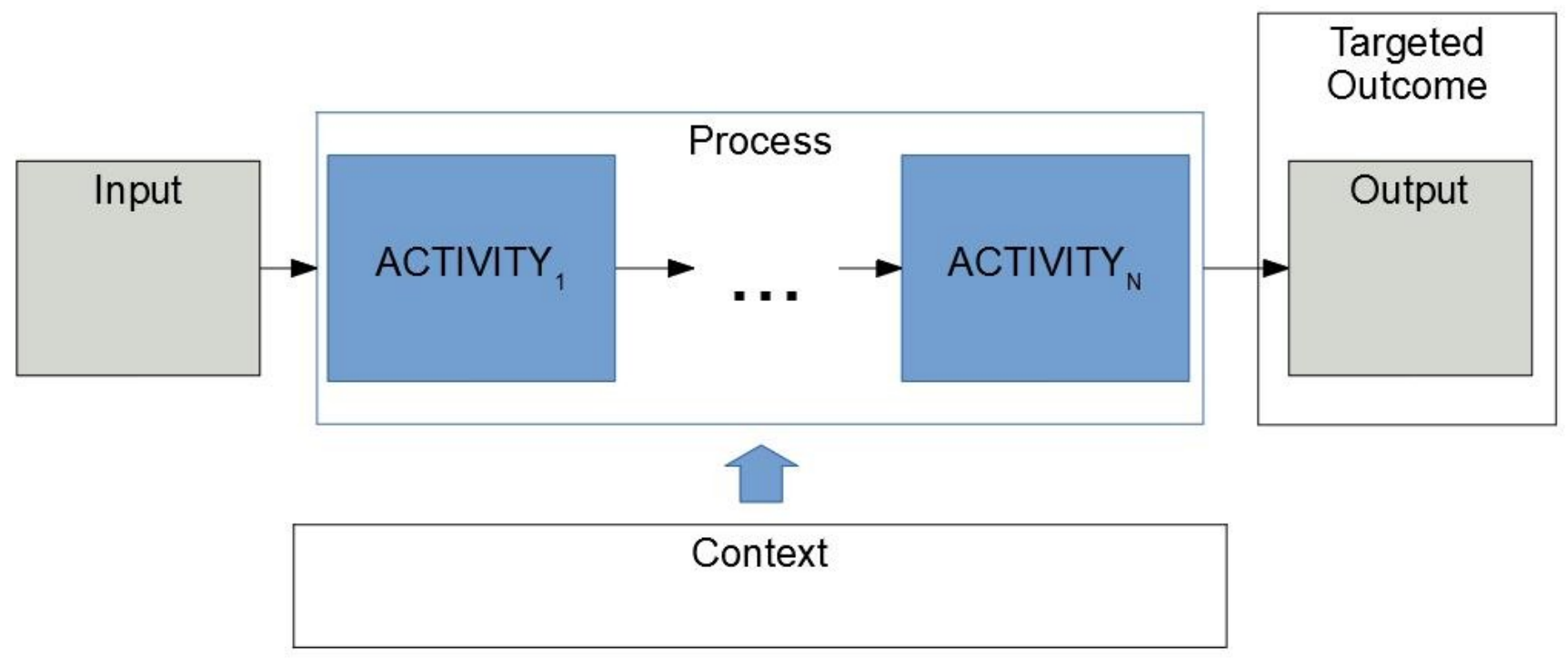

Figure 7: Defining simple functional models of physical or virtual manufacturing processes.

Processes are defined here as functions that can receive input multiple times within the same instance of execution.

\section{The Limits to Complexity in Manufacturing}

One active area of research in manufacturing is measuring the complexity of manufacturing processes, as well as relating that complexity to properties of those processes, like potential for error [2]-[7]. But what level of complexity in manufacturing processes is not yet possible?

Intuitively we know that current human manufacturing processes and manufacturing cooperation processes are not yet reliably capable of many outcomes nature can reliably accomplish, for example, manufacturing a wildebeest calf and synchronizing that process with the manufacture of hundreds of thousands of other wildebeest calves in the grasslands of the Masai Mara. Current human manufacturing is not reliably capable of creating manufacturing technology like the womb of that wildebeest's mother. The point is not to manufacture living things, the point is just to emphasize that there are entire classes of products and services that can't reliably be manufactured, because they are too complex, and the point is to understand in the simplest terms possible the source of that complexity and how to increase capacity to navigate it.

As discussed, GCI has been defined as a system with the potential to increase the general problemsolving ability of a group so that it is vastly greater than that of any individual in the group. In order to define the potential impact of increased problem-solving ability in objective terms, it is necessary to have a model for the complexity of both problems and solutions, as well as having a model for impact of solutions on a given problem. The Human Centric Functional Modeling approach in which the model of GCI discussed in this paper was defined represents any process as potentially being expressed in terms of Nth order interactions between component processes.

In the case of manufacturing, the outputs of processes are products. Products are then represented in terms of Nth order interactions between manufacturing processes, where $\mathrm{N}$ can be too high to be reliably achievable by any individual human cognition. What classes of processes require Nth order interactions in order to be reliably achievable, where $\mathrm{N}$ is high? What subset of those complex 
processes are processes to manufacture a product? What classes do those products that are too complex to manufacture fall into? What are the common features of the problems those products that are too complex to manufacture solve?

\begin{tabular}{|l|l|}
\hline Classes of Manufacturing Problems and Solutions Requiring General Collective Intelligence \\
\hline $\begin{array}{l}\text { Feature of Manufacturing Problem or } \\
\text { Solution }\end{array}$ & Nth Order Interaction Required \\
\hline $\begin{array}{l}\text { Self-Containment in Biological Machinery } \\
\text { (Biological machines that must produce some } \\
\text { functionality from higher order interactions } \\
\text { between the limited set of components they } \\
\text { contain, this includes self-reproduction, or self- } \\
\text { healing.) }\end{array}$ & $\begin{array}{l}\text { Search for manufacturing processes that produce a } \\
\text { functional component } \mathrm{P}_{\mathrm{i}} \text { of a product P. Search for } \\
\text { combinations of interactions between N of those } \\
\text { processes, where those interactions manufacture P } \\
\text { from its components } \mathrm{P}_{\mathrm{i}} \text {, and where N is increased to } \\
\text { the point that functional components can be reliably } \\
\text { manufactured through such interactions even if too } \\
\text { complex for individual cognition. }\end{array}$ \\
\hline
\end{tabular}

Table 1: Classes of design problems not reliably conceivable, and classes of design solutions not reliably solvable without $G C I$.

The list of manufacturing problems and solutions requiring collective intelligence in table 1 is not exhaustive, many more might exist.

\section{The Limits to Cooperation in Manufacturing}

Another active area of research in manufacturing is defining systems with the capacity to orchestrate complex cooperation[8]-[9]. But what level of complexity in manufacturing cooperation is not yet possible?

Increasing the complexity of structures that can be reliably designed or manufactured is one purpose of General Collective Intelligence. Another is facilitating the complex cooperation required to make those products or services sustainably viable. For example, currently existing patterns of human cooperation might not yet be reliably capable of creating ecosystems that make manufacturing any product or service sustainably viable in the USA. Manufacturing technology does not exist in a vacuum. Henry Ford's invention of the assembly line was one of history's most important manufacturing innovations. But without significant oil reserves and the US interstate highway system (still the largest project in human history to date at $\$ 500$ billion), that manufacturing innovation alone could not have driven such success in a company that produced cars. But cooperation with an infrastructure program requiring such a massive investment is not reliably possible for all of America's products and services.

A GCI defines cooperation in terms of Nth order barter transactions, or any other pattern that is most fit in achieving cooperation, so that opportunities for achieving benefit through cooperation can be reliably found algorithmically even where too complex for any individual human mind. Simple (first order) barter transactions in which one person exchanges something of equal value to another person are easy for people to negotiate. However, in an Nth order barter transaction the first person gives something to the second person, the second person gives something to the third person, and so forth until the Nth person give something to the first person. Even if all the participants were brought into a single arena, Nth order barter transactions might be too difficult for humans to detect. But for computers the task is simple. Such barter transactions can be among a network of peers (Nth order network barter transactions), or can be hierarchical (Nth-order hierarchical barter transaction). In an 
Nth order hierarchical barter transaction $\mathrm{N}$ people each give something to 1 person in return for something of $\mathrm{N}$ times that value which they share.

Individual human intelligence has the general problem-solving ability required to abstract the concept of value so that it's possible to see the value in creating any tool to achieve any purpose, and so it's possible to exchange and accumulate enough of that value for today's manufacturing processes to be possible. GCI on the other hand is predicted to create the additional problem-solving ability required to abstract the concept of abstracting concepts, so that it's possible to see the value in cooperating to define abstractions (like patterns of cooperation) to create tools to achieve any purpose, where those tools are not possible today. And so it's possible to exchange and accumulate enough of that value for the manufacturing processes of the future to be possible. Assume that through such patterns of cooperation a cognitive system (in this case a collective one) has the capacity to scale cooperation to execute reasoning processes to whatever order $\mathrm{N}$ is required to conceptualize manufacturing processes of the complexity required for a given manufacturing process. Assuming that Nth order cooperation requires GCI in order to be reliably achievable when $\mathrm{N}$ is sufficiently high, then any process requiring the capacity to scale cooperation to the Nth order then requires GCI to be reliably achievable.

Manufacturing in any particular geography is currently observed to be unstable in that it starts, stops, or moves according to factors like what is the lowest cost center globally. Nature on the other hand does not manufacture any of its products or services in isolation, but creates entire ecosystems within which manufacturing of a range of products is sustainably viable. Examples describing nature's manufacturing of living systems appear facetious, but they might be necessary since the theory of GCI suggests that no current human decision-making system aside from GCI has the capacity for such complex cooperation, and therefore manufacturing processes don't have the capacity for such complex cooperation without GCI. And since GCI has not yet been been implemented, the capacity for such complex adaptive manufacturing outside of nature cannot exist.

Nature, for example, did not create the process for manufacturing any given organism in isolation. Creation of that manufacturing process was accompanied by creation of an ecosystem (in the case of a specialized organism like the Orangutan, the rain forests of Borneo) to support the sustainable manufacture of that product (orangutans), so that the manufacturing process is stable enough not to be discontinued by any typical changes the environment might experience. By contrast, take any current human manufacturing process. That process is typically portable in that it is loosely coupled with it's environment and can occur wherever inputs can be shipped. The production of those inputs or of the manufactured product in a given environment are not coupled together in a way that makes them all sustainably viable. That manufacturing process therefore might suddenly switch to another location when some inputs become cheaper there. Without GCI, national organizations that invest in advanced manufacturing research then might actually be investing in the other nations that are the lowest global cost centers for manufacturing.

General Collective Intelligence is not only required for complex manufacturing and design. It is also required to create a stable local ecosystem that makes local manufacturing (and hence more robust supply chains) reliably viable. For all the above reasons, GCI is critical in creating an economic recovery plan using manufacturing to reliably drive economic recovery caused by disruption such as the COVID-19 pandemic [11]. 


\begin{tabular}{|l|l|}
\hline $\begin{array}{l}\text { Feature of Manufacturing Cooperation } \\
\text { Problem or Solution }\end{array}$ & Nth Order Cooperation Required \\
\hline $\begin{array}{l}\text { Sustainability (Sustainable machines that must } \\
\text { share a limited number of components across } \\
\text { enough other machines that maximizing value by } \\
\text { minimizing consumption is viable.) }\end{array}$ & $\begin{array}{l}\text { Coordinate the manufacture of N products, where N } \\
\text { is increased to the point that sustainability (rather } \\
\text { than planned obsolescence) is economically viable } \\
{[1] .}\end{array}$ \\
\hline
\end{tabular}

Table 2: Classes of design problems not reliably conceivable, and classes of design solutions not reliably solvable without $G C I$.

Again, the list of manufacturing cooperation problems and solutions requiring collective intelligence in table 2 is not exhaustive, many more might exist.

\section{The Impact of Removing the Limits to Complexity and Cooperation}

After functional models of all products and services created by the group have been defined, these functional models might eventually be aligned into a developmental hierarchy. If products $\mathrm{P}_{1 \mathrm{il}}$ to $\mathrm{P}_{1 \mathrm{iN}}$ or services $S_{1 i 1}$ to $S_{1 i N}$ are required as inputs to the manufacturing process for component $P_{1 i}$ of product $P_{1}$, and products $P_{j 1}$ to $P_{j M}$ are required as equipment, or services $S_{j 1}$ to $S j M$ being required as activities in the manufacturing process for component $\mathrm{P} 1 \mathrm{i}$ of product $\mathrm{P}_{1}$, then by defining $\mathrm{N}$ th order chains of cooperation between this matrix of products and services it might be possible to design all products and services such that they can be constructed from a basic set of self-contained set of functionality. Or in other words, from a basic set of building blocks. Such building blocks might be used to construct other building blocks (or if their functionality included replication, might construct those other building blocks themselves), that might construct other building blocks, that might eventually construct all existing products and services. Once all designs have been aligned into a single hierarchy, that can be constructed from a minimal set of functional components, then the set of all functional models of all designs for all products and services effectively forms a "collective genome". And the basic building blocks form "collective gametes".

With this collective genome capable of storing processes of higher order complexity (involving interactions between processes where those interactions require the ability to navigate higher levels of complexity than currently possible), and with these collective gametes capable of expressing those interactions from this stored information, then design and manufacturing processes might include interactions that are far higher order, and therefore far more complex than currently possible.

The categories of products too complex to manufacture currently include much biological machinery. For example, rather than manufacturing a calculator, through some complex manufacturing process one might grow one. The difference between growing and manufacturing a calculator is that the manufacturing processes must be contained within the interactions possible for the device. And the design for those manufacturing processes must be stored within the device itself. This requires the ability to search all higher order interactions until the required set is found.

Functional models of designs and manufacturing processes stored in a conceptual space format defined within an HCFM approach appear to be expressed as graphs with the potential to be independent of language, and so able to be understood by any language spoken by any civilization to come, without needing to leave behind any mapping to any third language that is known to the speakers of that future language (i.e. without a "rosetta stone" being required). Such functional models would have the effect of providing human civilization with far greater resilience to any catastrophic event, because society might be reconstructed even if computer systems or operating systems capable of reading whatever 
media format knowledge has been stored in are no longer in existence and can't be recreated before that knowledge is lost.

\section{Conclusion}

In summary, organizations like the NSF support cutting edge research in the US to create manufacturing and other technologies that can give US businesses competitive advantage. But with the instability of manufacturing location, any investment in manufacturing technology alone without investment in GCI as technology to create the ecosystem to support manufacturing locally, is an investment in China. In that respect, US government funded research is actually supporting Chinese commercial interests. At the same time, China's efforts to become the lowest manufacturing cost center might potentially be harmful for the people and environment of China itself. Creating the capacity for the next level of complexity in manufacturing, and creating the capacity for the next level of complexity in cooperation for complex manufacturing, so that far better manufacturing processes are available, and so that those processes can always reliably be used to stimulate local economic stability, might be advantageous to the economies of both countries.

\section{References}

[1] Andy E. Williams, General Collective Intelligence as the Emerging Paradigm in Human-Centric Design for Sustainability, accepted for publication in the proceedings of the peer-reviewed $26^{\text {th }}$ International Sustainable Development Research Society Conference that will take place the 15-17th of July, 2020 in Budapest, Hungary

[2] A Calinescu, J Efstathiou, J Schirn \& J Bermejo (1998) Applying and assessing two methods for measuring complexity in manufacturing, Journal of the Operational Research Society, 49:7, 723-733, DOI: $10.1057 /$ palgrave.jors.2600554

[3] Efthymiou K, Pagoropoulos A, Papakostas N, Mourtzis D. Manufacturing systems complexity review: Challenges and outlook. Procedia CIRP, 2012, vol.3(1), p.644-649.

[4] ElMaraghy W, ElMaraghy H, Tomiyama T, Monostori L. Complexity in engineering design and manufacturing. CIRP Annals - Manufacturing Technology, 2012, vol.61(2), p.793-814.

[5] Efthymiou K, Mourtzis D, Pagoropoulos A, Papakostas N, Chryssolouris G. Manufacturing systems complexity analysis methods review. International Journal of Computer Integrated Manufacturing, 2015, vol.29(9), p.1025-1044.

[6] O. Kuzgunkaya, H.A. ElMaraghy, Assessing the structural complexity of manufacturing systems configurations, International Journal of Flexible Manufacturing Systems, 18 (2) (2006), pp. 145-171 [7] Fan Guoliang, Li Aiping, Moroni Giovanni, Xu Liyun, Liu Xuemei, Operation-based Configuration Complexity Measurement for Manufacturing System, Procedia CIRP, Volume 63, 2017, Pages 645-650, ISSN 2212-8271, https://doi.org/10.1016/j.procir.2017.03.136. [8] Marie-Pierre Pacaux-Lemoine, Damien Trentesaux, Gabriel Zambrano Rey, Patrick Millot, Designing intelligent manufacturing systems through Human-Machine Cooperation principles: A human-centered approach, Computers \& Industrial Engineering, Volume 111, 2017, Pages 581-595, ISSN 0360-8352, https://doi.org/10.1016/j.cie.2017.05.014.

[9] Jinhua Zhou, Jianjun Zhu, Hehua Wang, Strategic cooperation with capital-constrained supplier and downstream competition in complex product systems, Computers \& Industrial Engineering, Volume 139, 2020, 106139, ISSN 0360-8352, https://doi.org/10.1016/j.cie.2019.106139.

[10] Patrik Grznár \& Martin Krajčovič \& Štefan Mozol \& Marek Schickerle \& Gabriela Gabajová \& Monika Bučková, 2019. "Cooperation Between Human And Agents In Holonic Manufacturing Systems," CBU International Conference Proceedings, ISE Research Institute, vol. 7(0), pages 111-117, September. 
[11] Williams, A. E. (2020, April 20). Transforming Economies and Generating Sustainable "Green" Economic Growth After the COVID-19 Pandemic through General Collective Intelligence.

https://doi.org/10.31235/osf.io/arw7c 